Euskal ikerketen aldizkaria | Revue d'études basques |

Revista de estudios vascos | Basque studies review

$6 \mid 2001$

Numéro VI

\title{
Note sur les phrases complexes à protase corrélative du basque
}

Georges Rebuschi

\section{OpenEdition}

Journals

Édition électronique

URL : http://journals.openedition.org/lapurdum/1232

DOI : 10.4000/lapurdum.1232

ISSN : 1965-0655

Éditeur

IKER

Édition imprimée

Date de publication : 1 octobre 2001

Pagination : 289-317

ISBN : 2-84127-156-0

ISSN : $1273-3830$

Référence électronique

Georges Rebuschi, « Note sur les phrases complexes à protase corrélative du basque », Lapurdum [En ligne], 6 | 2001, mis en ligne le 01 juin 2009, consulté le 30 janvier 2020. URL : http:// journals.openedition.org/lapurdum/1232 ; DOI : 10.4000/lapurdum.1232 


\title{
Note sur les phrases complexes à protase corrélative du basque
}

\author{
Georges Rebuschi \\ Sorbonne Nouvelle \& CNRS \\ (LACITO, \& associé à l'UMR 5478)
}

\section{Introduction}

L'objectif central de cet article-ci est le suivant ${ }^{1}$ : reprendre le $\S 241$ de la Grammaire [ ...] de Lafitte (1962, p. 105) consacré aux « relatifs indéfinis en ere » et à la structure des phrases dans lesquelles on les trouve, sur la base des acquis de la linguistique des vingt dernières années. Dans cette perspective, j'essaierai de monter en particulier que les « relatifs indéfinis en ere » ne sont en fait pas des relatifs - mais des éléments wh-non-relatifs, que la présence, dans la principale, d'un pronom résomptif appartenant « à la série [du démonstratif] hura » n'est pas un artifice stylistique typique de l'euskara, que le pronom haina que les « anciens » lui substituaient (parfois !) n'est pas un simple pronom, mais un élément quantificationnel, et enfin (et surtout) que la conjonction eta qui apparaît soit en tête de la principale, soit immédiatement après l'indéfini, n'est pas non plus un simple élément décoratif « renforçant » quoi que ce soit, mais une tête fonctionnelle qui est toujours présente, même si elle n'est pas réalisée, et qui contribue à

${ }^{1}$ Ce texte développe la deuxième partie de l'exposé que j'ai fait à Bayonne lors du Colloque international organisé, à l'occasion du centenaire de la naissance de Pierre Lafitte, par l'UMR IKER et l'Académie basque (Euskaltzaindia) en octobre 2001. La première partie, plus spécifiquement consacrée à la syntaxe des phrases complexes dans diverses langues, dont le basque bien entendu, doit être publiée dans les Actes de ce Colloque (Rebuschi, sous-presse) ; dans la mesure du possible, je tenterai d'éviter les répétitions, mais de légers recoupements sont inévitables.

Je souhaite remercier les participants à ce colloque pour leurs remarques et questions, ainsi qu'Alex Grosu, pour ses commentaires sur une version antérieure de ce texte, et André Rousseau pour nos échanges espacés mais, du moins en ce qui me concerne, très fructueux sur les rapports logiques entre corrélatives et conditionnelles.

Une version préliminaire de ce travail avait été présentée à la Table ronde internationale sur la syntaxe et la sémantique des relatives (Tel Aviv, juin 2000) : que les organisateurs et les participants à cette table ronde soient également remerciés.

Les abréviations suivantes seront utilisées : ACC, accusatif ; AUX, auxiliaire ; DAT, datif ; E, ergatif ; F, focus ; FEM, féminin ; FUT, futur (ou prospectif) ; GEN, génitif ; IMPER, impératif ; INT, interrogatif ; LOC, locatif ; PL, pluriel ; REL, relatif ; SUBJ, subjonctif. 
l'interprétation globale de la phrase complexe.

Si je vais donc être amené à prendre le contre-pied d'à peu près tout ce qui est dit dans cette section 241, ce n'est pas pour le simple plaisir d'effectuer un exercice d'application de la " philosophie du non » chère à Bachelard : on verra au contraire que ces résultats permettent d'unifier le traitement des phrases complexes conditionnelles et des phrases complexes à corrélatives, en ramenant les secondes à une variante morpho-syntaxique des premières - ce qui, si l'analyse est dans la bonne direction, n'est somme toute pas tout à fait trivial.

\section{Les phrases complexes à protase corrélative (PCPC) : éléments de description et problématique}

2.1. Il s'agit de constructions complexes comme celle de (1), où l'on a deux propositions ; la première (ou protase) contient un élément $W h$ - (dit $N Z$ en basque), ainsi qu'une marque de subordination (non-complétive) ; la raison pour laquelle j'évite d'employer l'expression « relatives libres » pour m'y référer apparaîtra dans les sections 3 et $4^{2}$. Crucial pour nous sera le fait que la seconde, l'apodose, contient (optionnellement) d'une part un pronom de rappel ou pronom corrélat (hura ci-dessous) et d'autre part qu'elle peut être introduite par le mot eta, qui est par ailleurs une banale conjonction de coordination.

(1) Nork ere huts eginen bait du, (eta) (hura) gaztigatua izanen da qui-E ere faute faire-FUT bait AUX et lui puni être-FUT AUX 'Celui qui commettra une faute sera puni' lit. 'Quiconque ${ }^{3}$ fera faute, (et) (lui) sera puni'

Ces constructions sont attestées dans tout le domaine oriental (sauf dans les documents de roncalais auxquels j'ai eu accès) depuis les premiers textes, qui datent du $16^{\mathrm{e}}$ siècle, jusqu'au $20^{\mathrm{e}}$ siècle ; la marque de subordination de la protase est (presque) toujours bait- dans les dialectes du Nord, et soit -(e)n, soit, plus rarement, bait- dans ceux parlés en Navarre. Par ex., dans la trad. de l'Evangile selon St. Jean par Joaquin Lizarraga d'Elkano, texte de la fin du $18^{\mathrm{e}}$ ou du début du $19^{\mathrm{e}}$, j'ai relevé quinze corrélatives en -(e)n, mais une seule en bait-, citée en (2) [Jn 14,21] ${ }^{4}$ :

${ }^{2}$ Les PCPC sont traitées comme des relatives d'un type particulier dans Euskaltzaindia (1999, chap. 5, 247-257).

${ }^{3}$ Se rappeler que ce mot s'écrivait en qui c'onques en ancien français, où l'on trouve, outre qui, un que subordonnant (noté $c^{\prime}$ ) et enfin onc, onques 'jamais', cf. l'anglais ever - sur le basque ere, que je ne traduis pas, voir la note 16 infra), puis le $\S 7.3$, sous (35).

${ }^{4}$ La proportion de préfixation en bait- de la forme verbale fléchie dans les relatives adnominales est par contre un peu plus grande. Il en va de même des corrélatives dans les divers sermons du même auteur, e.g. Urteko igande guzietarako prediku laburrak (ms. \pm 1800 , éd. de 1990, p. 156) : Ala nola bista labúr duéna baliatzen baita begiórdes edo anteójoes, billatuz árgi, ta beirátuz 
(2) Nork ere baititu ene manamenduak, ta guardátzen: ori dá onesten-nauéna. qui-E ere bait-les-a mes commandements et gardant celui-là est le-qui-m'aime lit. 'Quiconque a mes commandements et les retient, celui-là est celui qui m'aime.' [baititu $=$ bait ditu $]$

Ces phrases complexes sont aussi attestées dans les dialectes occidentaux, du moins dans leurs plus anciens monuments, cf. (3a) - où baist- est une variante de bait $^{5}$ - et (b), probablement déjà archaïsant - sinon, ce sont uniquement des constructions elliptiques, sans verbe fléchi et donc sans marque de subordination ou de dépendance dans la protase, qu'on y trouve, cf. (4) :

(3) [dialecte bizcayen]

a Zelan baista oiala alakoa mendela. comment baist-est le-tissu telle/de-cette-sorte la-lisière

'Comme est l'étoffe, ainsi (est) la lisière' (Refranes y Sentencias, 1596, n 20)

b Nortzuben pekatubak parkatu daikezubeza.n, parkatuko jakez, qui-PL-GEN péchés pardonné vous-les-leur[SUBJ]- $n$ pardonner-FUT AUX eta nortzubenak geratu daikezubeza.n geratuak izango dira. [= Jn 20,23] et qui-PL-GEN-PL laissé AUX[SUBJ]- $n$ laissés être-FUT AUX

'Leurs péchés seront remis à ceux à qui vous les remettrez, et ceux à qui vous les retiendrez, ils leur seront retenus.' (Astarloa 1816-18, cité in Arejita 1978: 135)

(4) [dialecte guipuzcoan]

Nolako elizalde, alako abade.

'Qual la ante iglesia, tal el abad'

(Isasti $\left.1625, \mathrm{n}^{\circ} 60\right)^{6}$

2.2. Il existe diverses possibilités d'analyse des PCPC, qui sont autant influencées par les langues spécifiques étudiées que par les préférences théoriques des auteurs qui ont effectué ces études. La position que j'adopte peut se résumer par les points suivants :

(5) a Tant l'existence d'un pronom corrélatif que la possibilité pour la protase d'exhiber deux expressions wh- interdit une analyse par mouvement.

b La conjonction qui introduit l'apodose est en fait une tête fonctionnelle conjonctive qui prend la protase comme spécificateur et l'apodose comme complément.

c La phrase complexe ainsi construite, qui semble hériter son statut énonciatif (sa « force » dans les termes de Chomsky et Rizzi) de l'apodose, la reçoit en fait d'une tête $\mathrm{C}^{\circ}$ qui prend la $\mathrm{PCPC}$ comme complément.

En ce qui concerne (5a), notons simplement ici que s'il y a deux expressions $w h$ - dans la protase, et donc deux pronoms corrélatifs dans l'apo-

óngi: alá ariman ezautzekó óngi dá eskátzea árgi Jangoikoai... 'De même que celui qui a la vue courte se sert de verres ou lunettes, trouvant [ainsi] la clarté et voyant bien, de même, pour connaître l'âme, il est bon de demander la lumière à Dieu.'

${ }^{5}$ Azkue (1923-25, p. 357), signale que cette variante était encore vivante dans certains parlers biscayens au début du $20^{\mathrm{e}}$ siècle.

${ }^{6}$ Voir aussi les num. 61-63, même page. Sur l'unique corrélative présente dans le catéchisme guipuzcoan d'Ubillos (1775), voir (24) infra et l'alinéa qui précède cet exemple. 
dose, il est tout simplement impossible de considérer que la première a été extraite de l'une de ces deux positions dans l'apodose, car (i) elle ne peut avoir été extraite des deux simultanément, et (ii) il faudrait reconnaître de toute manière que l'autre pronom n'est pas une trace « déguisée » en résomptif, mais bien un pronom généré dans la base dans cette position. En voici deux exemples bas-navarrais, le premier du $16^{\mathrm{e}}$ siècle, et le second du $20^{\mathrm{e}}$ :

(6) a B. d'Etxepare (1545, Doctrina Christiana, v. 14)

Nork zer hazi erein [baitu] biltzen dizi komunki.

qui-E quelle semence semé bait-AUX récoltant AUX communément

'On récolte ordinairement le grain qu'on a semé.'

[trad. de Lafon]

lit. 'Qui quel grain il sème, (il le) récolte communément.'

b Nork zertan baitu bere burua bilhatzen alabainan, qui-E en-quoi bait-AUX lui-même cherchant en-effet

hartan ere du hark bere amodioa galtzen.

(Léon 1929., p. 148: 3.5.6)

en-cela même AUX lui son amour perdant

'En effet, en quoi qu'on se recherche soi-même, en cela-même on perd son amour.'

De son côté, $(5 b)$ représente tout simplement l'hypothèse nulle, i.e. celle qui exige le moins de justifications indépendantes, du moins dans un cadre théorique qui admet les têtes fonctionnelles (" Gouvernement et liage » ou GB depuis Barriers, le Programme minimaliste ou PM chomskyen depuis une dizaine d'années, l'Antisymétrie de Kayne). En effet, même dans les modèles qui admettent plusieurs adjonctions, comme le PM, on voit mal comment mettre sur le même plan l'adjonction de la conjonction eta d'une part, et celle de la protase elle-même de l'autre ${ }^{7}$. De plus, rien ne justifierait l'ordre protase + conjonction plutôt que l'ordre inverse.

Quant à (5c), cf. Rebuschi (sous presse); il suffit de mentionner ici qu'une PCPC entière peut fonctionner comme une complétive, avant ou après des verbes comme erran 'dire' ou uste izan/ukan 'croire' : c'est alors le verbe fléchi de l'apodose qui porte le suffixe de subordination requis, -(e)la.

La problématique de l'interface entre la structure syntaxique et l'interprétation peut alors se concevoir de la manière suivante : quelles sont les contributions respectives (i) du pronom en $w h$-de la protase, (ii) de cette protase considérée globalement, (iii) de la conjonction, et (iv) du pronom corrélat contenu dans l'apodose ? Il va de soi qu'une démarche compositionnelle exige que l'on traite simultanément de toutes ces questions, ce que fort peu d'auteurs ont en fait tenté de faire jusqu'ici, se contentant généralement de ne traiter que de la valeur de la protase, ignorant le rôle de la conjonction (qui n'est pas toujours visible, ceci expliquant alors cela), et laissant à une

${ }^{7}$ Si le eta des PCPC basques devait être analysé comme étant adjoint à l'apodose dans une approche moins restrictive de la structure X-barre, les règles du jeu données par Kayne 1994 exigeraient par contre que cet élément se trouve alors dans la position de spécificateur d'une autre tête fonctionnelle, située plus bas que celle qui prend la protase pour spécificateur. En l'absence d'arguments allant dans ce sens, il est évidemment plus simple de faire de ces connecteurs non-standard les têtes elles-mêmes. 
théorie des pronoms résomptifs (toujours à construire) ou à une interprétation peu scrupuleuse des travaux d'Evans sur les pronoms dits « de type$\mathrm{E} »$ le soin de se charger de la non-contribution sémantique du corrélat, etc. Nous allons examiner ces questions dans les deux sections suivantes.

\section{Sur les protases corrélatives : nature et position de l'élément wh-}

3.1. La question de la structure interne des protases corrélatives et celle de la nature et de la position exactes de l'élément, ou des éléments, $W h$ qu'on y trouve sont intimement liées : s'agit-il de pronoms (ou adjectifs) interrogatifs, ou de relatifs, ou encore d'autre chose, et quelle est leur contribution à l'interprétation de cette subordonnée disloquée à gauche ?

La question de la nature des pronoms $w h$ - n'est pas oiseuse, parce que la sémantique de ces éléments n'est pas la même, et parce que, depuis le travail de Rizzi (1997) et bien d'autres à sa suite, on a de bonnes raisons de penser que la généralisation de Chomsky (1977) dans un article au titre explicite, 'On Wh-Movement', est partiellement une surgénéralisation, en ce sens que, syntaxiquement, les pronoms relatifs et les pronoms interrogatifs n'occupent pas la même position périphérique, les premiers se trouvant dans le spécificateur de la tête fonctionnelle la plus haute, et les seconds, dans celui d'une tête fonctionnelle inférieure, $\mathrm{cf} .(7)^{8}$ :

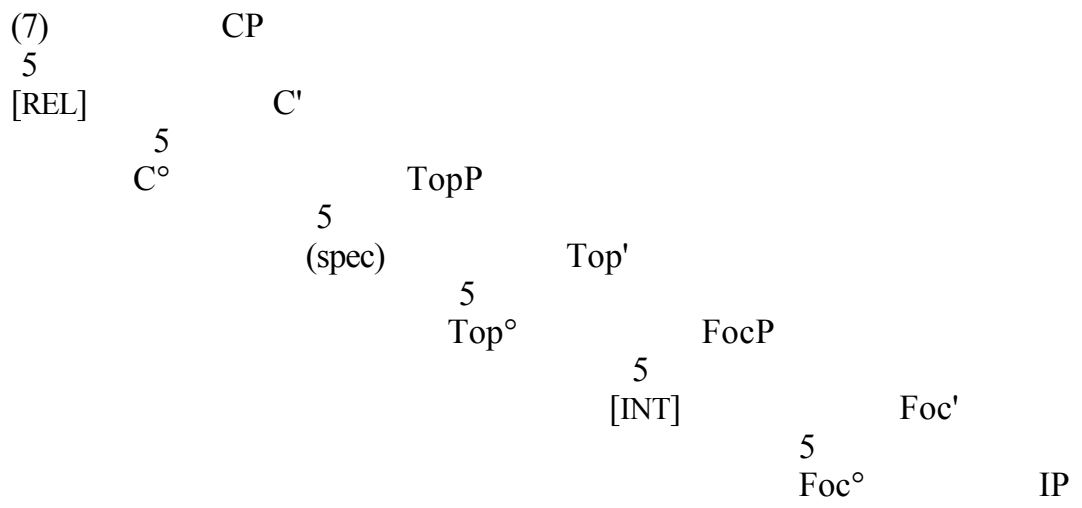

Peu importe en fait la nature exacte de la tête et de la projection intermédiaire. Ce qui compte, sur le plan empirique, ce sont les différences que l'on peut observer entre la position "REL" (pour relatif) et la position "INT" (pour interrogatif) dans cet arbre. De ce point de vue, le hongrois est une langue particulièrement significative, parce que, comme le montrent les ex. (8a,b) empruntés à Lipták (2000), cette langue possède les deux types de protases corrélatives.

${ }^{8}$ Rizzi (1997) propose aussi la présence d'une tête et d'une projection de Top. en-dessous de Foc., mais cela est sans importance en ce qui nous concerne. 
(8)
kér, az.t elve.het.i.
[Lipták 2000, ex. (1)]
qui[REL] quoi[REL,ACC] veut cela-ACC il-peut-le-prendre
'Everyone can take what he wants' / 'chacun peut prendre ce qu'il veut.' qui [INT] quoi [INT,ACC]
(id.)
b Ki mit kér, azt elveheti. [(ibid.), ex. (3)]

Cet auteur (dont l'analyse est indépendante du travail de Rizzi) indique crucialement à cet égard que les propriétés syntaxiques des deux types de protases ne sont pas les mêmes ; en particulier, une expression quantifiée et un XP focalisé peuvent suivre un $w h$-relatif, mais pas un $w h$-interrogatif, cf. $(9 a, b)[$ ses ex. $(5 a, b)]$ :

(9) a Aki amit tegnap PETERNEK adott, qui[REL] quoi $[\mathrm{REL}, \mathrm{ACC}]$ hier à-Pierre $[+\mathrm{F}]$ donna azt sosem látja vissza. cela-ACC jamais il-le-verra à-nouveau 'Ce que quiconque a donné hier à Pierre, jamais il ne le reverra.'

b * Ki mit... tegnap PETERNEK adott, ... qui[INT] quoi [INT,ACC]

3.2. En basque, il y a deux types d'arguments que l'on peut donner pour dire que les éléments $w h$ - des protases corrélatives ne sont pas des relatifs. D'une part, sur le plan lexical, le paradigme des interrogatifs et des relatifs (tels qu'ils étaient employés jusqu'au $19^{\mathrm{e}}$ siècle, puisqu'un opérateur phoniquement vide les a usuellement remplacés depuis) n'est pas strictement identique, car dans les interrogatives qui ne sont pas liées étroitement au contexte ou au « discours ", le pronom qui correspond à des référents humains était, et reste, nor (quoique, il est vrai, il tende à être remplacé par zein chez de nombreux locuteurs de guipuzcoan), tandis qu'à de très rares exceptions près, le pronom relatif pour les humains, aussi longtemps qu'il a été employé, était zein (ou zoin) ; dans le domaine des locatifs, de la même manière, lorsqu'il n'y a pas un ensemble de référence clos et de petite taille, l'interrogatif a toujours été non, nun, alors que le relatif était zeintan, zointan. Or, dans les protases corrélatives, et, à nouveau, lorsqu'il n'est pas fait référence à un domaine connu et réduit, ce sont les mots nor et non qui sont employés. (Signalons aussi qu'Altube $(1929$, p. 139-140) proposait déjà explicitement un rapprochement entre les structures qui nous intéressent et des enchaînements de questions et de réponses.)

D'autre part, syntaxiquement, le basque offre des exemples analogues à ceux que Lipták cite pour les corrélatives à interrogatif en hongrois, cf. par ex. (10) avec un quantificateur universel distributif qui précède l'élément wh/NZ dans la protase (ce qui semble indiquer, il faut le remarquer, qu'une tête et une projection de quantifieur doivent s'intercaler au-dessus de la tête et de la projection de Focus - si c'est bien là la projection dont la position de spécificateur accueille l'élément $w h$-) :

(10) Nor-bera ZONBAT ere baita oldartzerat uzkurrago, chacun combien ere bait-est à-s'attaquer plus-veule hanbatez da egunetik egunerat ahulago...

d'autant il-est du-jour au-jour plus-faible

'Plus on met de de langueur à le repousser [=le mal], plus on s'affaiblit de jour en jour...' 
Le système de Rizzi, cf. (7), prévoit aussi qu'un topique puisse précéder un interrogatif, mais pas un relatif - et les données de Lipták le confirment. Or l'exemple (11) nous donne exactement cela : un XP topicalisé à gauche d'un syntagme en $w h$ -

(11) Bertutean NOR ere baita barnago sartzen, [L. Léon (id., p. 127, 2.12.7)] dans-la-vertu qui ere bait- AUX plus-dedans entrant hak berak maiz kurutze dorpeagoak ditu kausitzen. celui-là-E même-E souvent croix lourdes AUX trouvant 'Souvent, plus un homme pénètre dans la vertu, plus il trouve ses croix pesantes.'

Il parait donc bien établi que les éléments $w h$ - des corrélatives basques ne sont des relatifs $n i$ du point de vue lexico-morphologique, $n i$ du point de vue syntaxique (sur le rôle sémantique de maiz 'souvent', cf. 4.2.4 infra). La question se pose donc de savoir s'il y a des corrélats sémantiques à cette conclusion.

\section{Aspects sémantiques de la construction}

4.1. En fait, dire que nor ou zer (et les autres éléments $w h$ - du basque) dans les protases corrélatives n'occupent pas la position d'un relatif ne veut pas nécessairement dire qu'ils n'en ont pas l'interprétation dans le contexte qui nous intéresse, de même que dire qu'ils se rapprochent morpho-lexicalement et distributionnellement des interrogatifs ne signifie pas qu'ils en ont la sémantique (quelle qu'elle puisse être). Par ailleurs, comme on l'a dit plus haut, cela n'aurait aucun sens de se demander quelle contribution sémantique ces éléments apportent sans tenir compte en même temps de la structure globale de la phrase complexe dans laquelle ils se trouvent, et de la contribution sémantique individuelle des autres éléments pertinents.

De ce point de vue, on peut construire le problème comme suit : si un élément $w h$ - est extrait d'une proposition (disons d'un IP pour faire simple, mais on pourrait aussi bien dire ici d'un TP ou d'un AgrSP), au niveau interprétatif, sa trace sera traitée comme une variable. En d'autres termes, la traduction de l'IP en question sera ce qu'on appelle une proposition ouverte (angl. open proposition), c'est-à-dire un objet sémantiquement mal formé.

Or il existe trois types d'opérateurs qui peuvent, en liant la variable libre, transformer une proposition ouverte en une expression bien formée. Si donc nous trouvons parmi ces opérateurs celui qui, en harmonie avec la contribution des autres éléments pertinents de la phrase complexe prise dans sa totalité, peut lier correctement la variable, alors nous pourrons dire que cet opérateur est la traduction du wh- qui nous intéresse. Nous allons donc considérer ces opérateurs un à un, et chercher à voir dans quelle mesure ils sont compatibles (ou non) avec certaines données (plus ou moins) spécifiques au basque.

4.2. Tout d'abord, il y a les quantificateurs, qu'ils soient standard (comme tout ou il existe au moins un ... ) ou non (comme beaucoup, peu, un peu plus de $25,8 \%$ etc.) : en étant préfixés à une proposition ouverte, ils fournissent avec celle-ci une proposition quantifiée close, et donc suscep- 
tible d'être (in-)validée.

4.2.1. Dans un travail que j'avoue trouver discutable aujourd'hui (Rebuschi 1999), j'avais cru pouvoir, sur une base translinguistique relativement large, proposer que les langues à corrélatives dans lesquelles les éléments $w h$ sont des interrogatifs, et non des relatifs, sont aussi des langues dans lesquelles ces corrélatives s'interprètent de façon au moins non-marquée comme universelles ou génériques, alors qu'à l'inverse, en hindi (ou en latin, qui employait qui et non quis pour les humains), c'est-à-dire dans les langues qui emploient des relatifs lexicalement ou morphologiquement au moins parfois distincts des interrogatifs, c'est l'interprétation des protases comme des relatives restrictives qui est la plus normale - avec de simples extensions vers le sens des relatives libres.

Si ce fait n'est pas absolument faux, et si la formulation adoptée ici permet l'existence de langues à structures corrélatives des deux types avec ambiguiité (comme en hongrois supra) ou sans ambiguïté, comme en allemand, cf. $(12 \mathrm{a}, \mathrm{b})$ ci-dessous ${ }^{9}$, il y a des arguments de taille qui s'y opposent.

(12) a Wer Ohren hat zu hören, der höre !

Qui[INT] oreilles a pour entendre celui-là entende

'Que celui [quel qu'il soit] qui a des oreilles pour entendre entende !'

b Der die Hand mit mir in die Schüssel getaucht hat,

qui[REL] la main avec moi dans le plat trempé (aur)a der wird mich verraten.

(Mt 26,2)

celui-là AUX me trahira

'Celui [ + spécifique=la personne particulière] qui aura trempé sa main avec moi dans le plat, c'est lui qui me trahira.'

4.2.2. Ainsi, d'un côté, du point de vue strictement théorique, on doit se demander comment un quantificateur par exemple universel (ou encore générique) pourrait s'extraire de la proposition (syntaxique) de statut subordonné, et prendre l'ensemble de la structure complexe, bi-propositionnelle, sous sa portée. Noter de ce point de vue que les expressions universellement quantifiées de (13) n'ont précisément pas une grande portée :

(13) a Si tout le monde te critique, alors tu pourras être malheureux.

b Si tu trouves toutes les réponses, tu gagneras 10 euros.

En effet, dans de tels exemples, le quantificateur de la protase conditionnante ne prend pas l'ensemble de la phrase complexe sous sa portée ; ainsi, les conditions de vérité de (13b) sont radicalement différentes de celles d'une phrase où la quantification universelle aura la portée la plus grande, comme : Pout toute réponse $\boldsymbol{x}$ [= pour quelque réponse $\boldsymbol{x}$ que ce soit], si tu trouves $\boldsymbol{x}$, tu gagneras 10 euros. Par parité de raisonnement, on voit mal comment une protase corrélative dont l'élément $w h$ - serait de manière inhérente générique ou universel pourrait quantifier la PCPC entière.

${ }^{9}$ Ex. tirés de Heilige Schrift, Genève, Genfer BibelGesellschaft (1985). Ajoutons que l'emploi de der dans la protase est aujourd'hui archaïsant. 
4.2.3. Un second problème est lié à l'interprétation du pronom de reprise ou corrélatif, cf. hura (parmi d'autres, en basque), tot (en russe), der (en allemand), us (en hindi), etc. Il est vrai qu'il existe des théories sémantiques qui traitent parfois les pronoms de manière non-compositionnelle (la DRT), mais ces théories sont construites précisément pour rendre compte de phénomènes qui vont au-delà de la phrase, même complexe, ou encore ne tiennent pas compte du tout de cette notion ${ }^{10}$. Mais s'il est possible de traiter parfois les pronoms corrélats comme de pures variables, cela ne paraît absolument pas possible lorsque ces pronoms sont emphatiques, comme la forme complexe hak berak dans (11) supra ${ }^{11}$, ou encore lorsqu'ils sont remplacés par ce que j'ai cru pouvoir analyser comme un quantificateur universel "déguisé" en pronom (et alors nécessairement associé dans son interprétation à une position vide), haina (cf. Rebuschi 1997, 1998).

Observons ainsi certaines de ses propriétés. On le trouve (entre autres contextes) dans des phrases du type de (14), qui ne diffèrent de (1) que par le pronom en question :

(14) Nork ere huts eginen bait du, (eta) haina gaztigatua izanen da qui-E ere faute faire-FUT bait AUX, et lui puni être-FUT AUX 'Quiconque commettra une faute sera puni'

Il faut en particulier noter que haina, attesté dans les textes labourdins depuis le $16^{\mathrm{e}}$ siècle jusqu'au $19^{\mathrm{e}}$, ne se substituait jamais aux pronoms personnels de $3^{\mathrm{e}}$ personne (en fait, des démonstratifs, comme hura), lorsqu'il reprenait une relative " semi-libre ${ }^{12}$ disloquée et explicitement quantifiée par $g u z(t) i a(k)$ ou oro 'tout/tous', cf. $(15 \mathrm{a}-\mathrm{c})^{13}$ :

${ }^{10}$ Ceci n'est pas une critique, car il faut bien rendre compte du fait que certains « textes » comportant plusieurs phrases syntaxiques autonomes concaténées peuvent induire les mêmes interprétations, ou avoir les mêmes conditions de vérité, que des phrases complexes, ce qu'illustre le parallélisme entre (i) et (i') d'une part, et (ii) et (ii') de l'autre :

(i) Un homme entra. Il commanda une bière.

(i') Un homme entra, et il commanda une bière.

(ii) Tous les hommes sont mortels. Or Socrate est un homme. Donc Socrate est mortel.

(ii') Socrate étant un homme et tous les hommes étant mortels, Socrate est mortel.

Cela dit, dans la mesure même où les langues varient (au moins " superficiellement ») dans leurs structures morpho-syntaxiques, l'identification des phrases radicales (indépendantes) comme un domaine pertinent pour la linguistique proprement dite me paraît rester fondamentalement correcte.

${ }^{11}$ En effet, tout emploi emphatique ouvre au moins potentiellement la porte à une interprétation quantificationnelle du type : 'celui-là ET NUL AUTRE'.

${ }^{12}$ Les relatives « semi-libres » sont des structures que l'on peut traduire par des relatives libres (en quiconque en français, whoever en anglais, etc...) mais qui présentent crucialement un déterminant à droite $\mathrm{du} \mathrm{C}^{\circ}-(e) n$, comme le montre le SN disloqué de (15a).

${ }^{13}$ Avec des référents [+hn], les construction avec $g u z(t) i a$ au singulier 
(15) a huts egiten du.en.a, hura/haina gaztigatua izanen da faute faisant il-1'a-C ${ }^{\circ}$-DET lui $h$. puni être-FUT AUX lit. 'le qui [=celui qui] fera une faute, celui-là sera puni' [cf. (1)]

b huts egiten du.en guz(t)i.a, hura/*haina... faute faisant il-1'a-C ${ }^{\circ}$ tout-DET lui $h$. lit. 'tout le qui fait...'

c huts egiten du.te.n guz(t)i.ak, hek/*hainak... faute faisant ils-l'ont- $\mathrm{C}^{\circ}$ tout-PL eux haina-PL lit. 'tous les qui font...'

Par ailleurs, haina est totalement inattesté dans les phrases soumises à quantification adverbiale (comme, à nouveau, dans (11) supra, où figure dans l'apodose l'adverbe maiz 'souvent' — j'y reviens en 4.2.4 ci-dessous).

Voici pour finir un argument peut-être plus anecdotique (il ne figure pas dans Rebuschi $(1997,1998)$ ), mais qui est cependant, à mon avis, significatif en ce qui concerne la contribution sémantique de haina à la phrase dans laquelle il apparaît : dans les trois traductions labourdines de la période pertinente, à savoir, celles de Haraneder (1740), de Harriet (1855) et Duvoisin (1859-65), qui ne se correspondent mot-à-mot que très rarement, les trois textes emploient le pronom haina pour rendre le omnis latin de $\mathrm{Mt}$ 19,29, comme indiqué en (16).

(16) Mt 19,29 Et omnis, qui reliquerit domum, vel fratres [...] propter nomen meum, centuplum accipiet $[\ldots]$ 'Et quiconque délaissera sa maison ou ses frères $[. .$.$] pour$ moi (en) recevra le centuple.'

a Eta nork ere utziko bai[t]ditu bere etxea edo bere anaiak [...] ene izena gatik, hainak izanen duela ehunetan hainbertze.

b Eta nork ere utziko baititu bere etxea, edo bere anaiak [...] ene izena gatik, hainari ordain emanen zaio ehunetan bertzen hainbertze [...] (Harriet 1855)

c Eta nork ere ene izenaren ariaz utziko baititu etxea, edo anaiak [...], hainak ehunkun izanen [...] duela.

(Duvoisin 1859-65)

4.2.4. Un autre argument qui va contre l'hypothèse d'une quantification universelle inhérente des protases corrélatives basques est fourni par la quantification adverbiale (ou " non-sélective ») à laquelle je viens de faire allusion à propos de (11), et qui est naturelle dans cette langue dans les contextes qui nous préoccupent. Considérons de ce point de vue les trois traductions suivantes, qui s'étalent sur trois siècles maintenant, d'un même passage de l'Imitation, déjà citée, de Thomas A Kempis (3.12.4) ${ }^{14}$ (voir aussi (6a) supra, qui date du $16^{\mathrm{e}}$ siècle, et qui contient l'adverbe komunki) :

(17) a Nondik ere hartzen baitute atsegin, d'où ere prenant bait--AUX plaisir hainitzetan handik izaiten dute $\min$ eta dolore. souvent de-là ayant AUX peine et douleur (Pouvreau, 1669) lit. 'D'où (qu')ils tirent leur plaisir, souvent, (c'est) de là (qu')ils ont leur peine et leur douleur.'

sont aujourd'hui archaïques.

${ }^{14}$ Je n'ai malheureusement pas pu accéder à l'original latin. 
b Maiz zerk ere egin baitu hekien bozkariorik handiena, souvent quoi-E ere fait bait-AUX leur joie la-plus-grande hark kausatzen ere du hekien dolorerik minena. cela-E causant aussi AUX leur douleur la-pire lit. 'Souvent, quoi que ce soit qui leur donne leur plus grande joie, (c'est) cela (qui) leur cause aussi leur plus grande douleur.'

c Zertan ere baitute kausitzen atsegin, hartan ere dute en-quoi ere bait-AUX trouvant plaisir en-cela aussi AUX ardura nasaiki biltzen bihozmin. souvent abondamment recueillant douleur-morale lit. 'En quoi que ce soit qu'ils trouvent leur plaisir, (c'est) aussi en cela (qu')ils récoltent souvent (un) chagrin abondant.'

S'il est intéressant de constater que ce ne sont pas les mêmes arguments ou adjoints adverbiaux qui font l'objet du dédoublement corrélatif, il faut surtout noter que l'interprétation naturelle ici de souvent n'est pas une interprétation qui revient à quantifier des moments, ou des occurrences d'événements, mais une interprétation dite non-sélective telle que ces phrases reviennent à dire qu'il est vrai de beaucoup de gens qu'ils trouvent leur pire chagrin dans cela même où ils avaient trouvé trouvé leur joie la plus grande.

Si, d'une manière ou d'une autre, l'élément $w h$ - initial des protases corrélatives ci-dessus devait déclencher une quantification universelle ou générique pour ces phrases complexes, on serait cependant forcé de se rabattre sur la quantification adverbiale littérale, car il est impossible de quantifier deux fois les mêmes variables (ou encore de laisser un quantificateur sans variable à lier, cf. le principe chomskyen dit de « Pleine interprétation », repris quelques années plus tard par les sémanticiens sous le slogan « Il est interdit de quantifier à vide »). Or si l'interprétation selon laquelle 'il est vrai de tout individu qu'il trouve souvent sa plus grande peine dans ce dans quoi il avait auparavant trouvé sa plus grande joie' n'est pas à strictement parler impossible, elle est très peu naturelle.

De ce point de vue, on peut aussi réexaminer l'ex. (11) supra, qui pose, de manière peut-être plus aiguë encore, le même problème, car on a du mal à imaginer que cette phrase veuille dire qu'un individu quelconque pénètre, sorte, et entre à nouveau, mais plus profondément, dans la vertu, et qu'il trouve alors souvent que sa croix est plus dure à porter : à nouveau, maiz ne quantifie pas les occurrences d'événements, mais les individus concernés ${ }^{15}$.

4.3. Le second type d'objet formel qui permet de clore une proposition ouverte est l'opérateur iota de Russell, éventuellement revu à la lumière de la méréologie de Lesniewki. L'objet ainsi obtenu, au niveau du CP, n'est alors plus une proposition, mais une entité ou un quantificateur généralisé, quelque chose comme : l'unique individu (maximal) qui a la propriété $P$. La question de l'interprétabilité de la phrase complexe devient alors au moins

${ }^{15}$ En ce qui concerne le komunki de (6a), il a d'autres incidences encore, car il interagit de manière un peu surprenante avec l'effet quantificationnel normalement induit par la présence de deux éléments $w h$ - dans des structures de ce type (cf. aussi les interrogatives à $w h$ - multiple). 
aussi compliquée que dans le cas précédent, en particulier lorsqu'une quantification générique ou universelle est donnée par le pronom corrélat de l'apodose, cf. (16), ou dans le cas d'une quantification non-sélective à partir d'un adverbe par ailleurs temporel comme maiz, ardura, hainitzetan dans (17). Notons par ailleurs que personne n'a jamais proposé qu'un opérateur (syntaxique) $w h$-se traduise par un opérateur (logico-sémantique) iota : lorsque Srivastav (1991) a proposé d'interpréter globalement les protases corrélatives comme des individus (pluriels) maximaux, elle a en effet eu recours à un changement de type dans la composante sémantique de sa description, se contentant de traiter la relative appositive comme une relative intersective ordinaire, c'est-à-dire se traduisant par une propriété (voir 4.4. ci-dessous). De plus, ce changement de type (réinterprété par Grosu \& Landman (1998) comme une opération de maximalisation), dont je ne m'aventurerai pas à dire qu'il n'a pas lieu d'être postulé en hindi, est tout à fait exclu en basque pour les raisons quantificationnelles qui viennent d'être exposées ${ }^{16}$.

4.4. Le troisième moyen de faire d'une proposition ouverte une expression bien formée est de la préfixer d'un opérateur lambda qui lierait la variable libre. L'objet résultant est alors une propriété (ou, en extension, un ensemble d'individus ou d'objets qui se différencient de tous les autres par la possession de cette propriété). Dans le cas où les protases corrélatives s'interprètent comme des relatives restrictives, et où l'élément $w h$ - est effectivement un pronom relatif, il n'y a aucune difficulté : il y a plus de 25 ans déjà que B. Partee proposait que la contribution sémantique d'un pronom relatif est précisément celle d'un opérateur lambda (Partee 1975) ; si donc la proposition ouverte correspond à IP, l'expression-lambda entière correspondra au CP, l'élément $w h$ - se traduisant, tout simplement, par l'opérateur $\square^{17}$ :

${ }^{16}$ Ces mêmes raisons me poussent à considérer que, depuis les premiers textes connus, la particule ere qui accompagne souvent (mais pas toujours : voir (3) ou (4) par ex.) l'élément $w h$ - des protases corrélatives, et qui se traduit dans d'autres contextes par 'même' ou 'aussi', est, dans les phrases qui nous concernent, vide de sens, c'est-à-dire qu'elle ne peut pas s'interpréter comme le ever des mots anglais whoever, whatever (ou du onque de quiconque, cf. la note 3 plus haut) qui sont, eux, incompatibles avec toute forme de quantification adverbiale ou non-sélective. Pour une réinterprétation radicale, mais très programmatique, de ere, voir la fin de 6.1.

Pour en revenir à l'aspect quantificationnel des choses, je ne me prononcerai pas sur la maximalisation supposée par Grosu \& Landman (op. cit.) à propos des relatives corrélatives du hindi, mais soulignerai qu'il sans doute prématuré de généraliser les descriptions spécifiques de certaines langues ou certains groupes de langues à la « grammaire universelle » - cf. aussi Grosu (2000, p. 102) pour qui « une configuration avec adjonction à gauche [d'une relative libre] bloque toute autre interprétation que celle de la maximalisation ».

${ }^{17}$ Remarquer cependant que lorsque l'élément $w h$ - est phoniquement réalisée (au lieu d'être un « opérateur abstrait»), il est normalement accompagné d'une caractérisation du domaine concerné ([ \pm humain], par ex., cf. l'anglais who $\approx$ which), manifestant ainsi une propriété très générale des opérateurs syntaxiques des langues naturelles. 
(18) $\quad$ a $\quad\left[\mathrm{CP} \mathrm{WH}_{-i} \mathrm{C}^{\circ}\left[\mathrm{IP} \ldots t_{i} \ldots\right]\right]$

$\mathrm{b} \quad\left[\begin{array}{lll}\mathrm{a} & {[\mathrm{Q}(x)}\end{array}\right]$

Il suivrait de cette approche que la corrélative, interprétée comme une propriété, lierait une variable de propriété contenue dans la traduction ou interprétation du pronom corrélat : c'est la solution adoptée dans Rebuschi (1997, 1998).

Mais on a vu plus haut que les $w h$ - basques ne sont pas syntaxiquement des relatifs. Par ailleurs, même si les constructions corrélatives basques sont parfois (quoique rarement, en fait) employées pour faire référence à des individus spécifiques (au sens pragmatique ou énonciatif, c'est-à-dire à des individus connus du locuteur mais non de l'interlocuteur), je ne connais aucun usage strictement restrictif ou intersectif des protases corrélatives en basque ; or si les PCPC se traduisaient directement comme des expressions lambda et donc indiquaient des propriétés, on ne voit pas pourquoi elles ne pourraient pas servir à construire des relatives restrictives.

\section{Vers une solution}

Il faut donc ou bien se résoudre à une certaine distortion entre les données syntaxiques et les données sémantiques (ce que, pour des raisons méthodologiques, je préfère considérer comme un pis-aller, ou un constat d'échec, au moins temporaire), ou bien considérer qu'aucune des solutions envisagées à l'instant n'est satisfaisante.

5.1. Je souhaiterais donc terminer ce travail en proposant une autre approche, qui est au moins aussi compatible avec les données que l'hypothèse d'un wh-non-relatif qui serait malgré tout, sémantiquement, un opérateur lambda : le mot en wh-n'aurait simplement aucune propriété sémantique $^{18}$. Il aurait simplement le trait [+wh-] requis pour son mouvement, et sa fonction serait donc tout simplement de laisser une trace qui serait forcément interprétée comme une variable libre. Dans ces conditions, le liage non-sélectif de cette dernière par un adverbe explicite (comme maiz, etc., 'souvent') ou implicite, mais relié au temps générique de l'apodose (la proposition principale) serait tout à fait naturel et automatique. La trace des $w h$ déplacés fonctionnerait donc exactement comme les indéfinis tels qu'ils ont été réanalysés dans divers travaux plus ou moins récents, travaux selon lesquels, contrairement à l'analyse usuelle, un indéfini comme quelqu'un dans $(19 \mathrm{a}, \mathrm{b})$ ci-dessous n'est pas une expression quantifiée, mais précisément une variable libre dans la proposition minimale qui la contient, et à nouveau liée adverbialement ou génériquement par un opérateur issu de l'apodose (la proposition " conditionnée ») et ayant portée sur la phrase complexe dans sa totalité $^{19}$ :

${ }^{18}$ Voir Cheng (1995) pour des arguments (évidemment) tout à fait différents qui conduisent à la même conclusion en ce qui concerne le chinois.

${ }^{19}$ L'apostrophe, ici comme infra, signale des constantes non-logiques. 
(19) a Si quelqu'un parle, Pierre l'écoute souvent. $\operatorname{BCP}_{x}\left[\right.$ humain' $(x) \&$ parle' $(x)$ : écoute' $\left.\left(\mathrm{p}^{\prime}, x\right)\right]$

b Si quelqu'un parle, Pierre l'écoute. $\operatorname{GEN}_{x}$ [humain' $(x) \&$ parle'$^{\prime}(x)$ : écoute' $\left.\left(\mathrm{p}^{\prime}, x\right)\right]$

5.2. De ce point de vue, la parenté très étroite et évidente que le basque (parmi bien d'autres langues naturelles) offre entre les indéfinis et les wh- devient elle-même tout aussi naturelle, cf. les deux premières colonnes de (20) :

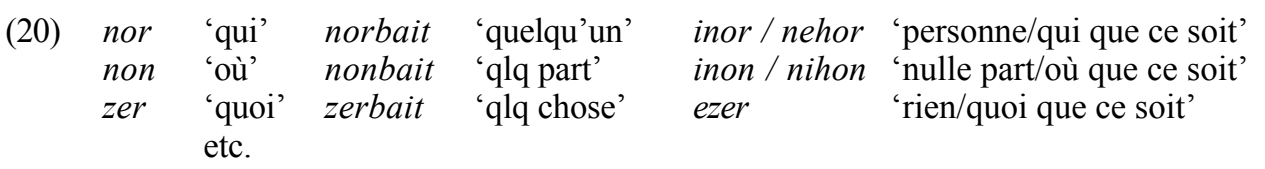

Ce n'est évidemment pas un hasard si l'on retrouve le suffixe baitdans la deuxième colonne : j'y reviens bientôt. Mais il y a plus. Du point de vue de l'interprétation des phrases complexes qui nous intéressent, il est clair qu'il y a une relation de paraphrase étroite entre les deux variantes de (21) :

(21) a Norbaitek huts egiten $\boldsymbol{b a}$-du, (orduan) (hura) gaztigatua izanen da. quelqu'un-E faute faisant si-AUX alors celui-là puni être-FUT AUX 'Si quelqu'un commet une faute, il sera puni.'

b Nork ere huts eginen bait du, (eta) (hura) gaztigatua izanen da. qui-E ere faute faire-FUT bait-AUX et lui puni être-FUT AUX 'Celui qui commettra une faute sera puni.' lit. 'Qui que/qui fera faute, et lui sera puni.'

Dans les deux cas en effet, on peut considérer qu'un opérateur de généricité lie deux occurrences d'une même variable, comme dans (22) :

$$
\mathrm{GEN}_{x}\left[\mathrm{P}^{\prime}(x): \mathrm{Q}^{\prime}(x)\right]
$$

où $\mathrm{P}^{\prime}$ représente le prédicat de la protase, $\mathrm{Q}$ ' celui de l'apodose, et les deux points entre les deux propositions, le connecteur non-standard, puisque la quantification n'est ni universelle ni existentielle - si elle était universelle, comme l'adverbe beti (ere) 'toujours', ou le pronom haina, cf. les ex. de (16), l'imposeraient, on aurait la variante (23) :

$$
\square_{x}\left[\mathrm{P}^{\prime}(x) \square \mathrm{Q}^{\prime}(x)\right]
$$

De ce point de vue, en ce qui concerne les pronoms corrélats, on peut donc proposer que si les pronoms non-emphatiques (y-compris pro) se traduisent bien, dans les contextes pertinents, comme des variables liées par un opérateur non-sélectif ou adverbial, dans les autres cas, il faut en faire une analyse complexe, le pronom haina ou les emphatiques comme (hura) bera 'celui-là et nul autre' (cf. l'ex. (11) et la note 11) apportant une force quantificationnelle propre à la phrase complexe prise dans sa globalité.

5.3. A observer soigneusement les rapports entre (21a) et (b), on voit que le choix lexical du connecteur linguistique (i.e. de la conjonction) qui unit 
les deux propositions syntaxiques est corrélé à la tête du premier $\mathrm{CP}$, i.e. de la protase, ce qui est un phénomène bien connu sous le nom d'accord entre spécifieur et tête: on a la paire ba- et orduan d'une part, et la paire bait- et eta de l'autre. Techniquement, cet accord se décompose plus précisément entre (i) une percolation du trait distinctif, quel qu'il soit - mais qui serait non-interprétable si mon analyse est corrrecte - qui permet d'opposer les têtes $b a$ - et bait- de la protase, vers la projection maximale CP de cette même proposition, et (ii) un " accord » entre la tete de la construction complexe (la conjonction, orduan ou eta) et la propriété que le CP qui occupe son spécificateur a ainsi héritée de sa propre tête - pour des arguments en faveur de l'hypothèse que ces conjonctions sont bien des têtes fonctionnelles, cf. Rebuschi (sous-presse).

Qui plus est, à l'intérieur même de la protase, on a aussi une forme d'accord entre la tête et le spécifieur, puisque, on y revient enfin, l'élément bait- figure ou bien sous $\mathrm{C}^{\circ}$, ou bien sur le pronom dont la position argumentale est interprétée comme une variable.

Mais il y a plus. Dans divers textes, j'ai relevé la présence de $b a$ - là où l'on attendrait bait-. Le premier dans l'ordre chronologique pose aussi un problème d'emprunts entre dialectes, car, comme l'a fait observer P. Altuna, une structure corrélative comme (24), qui contient un verbe fléchi, n'est pas usitée en guipuzcoan (voir (5) et les commentaires qui s'y rapportent). Les seconds exemples sont en baztanais du $19^{\mathrm{e}}$ siècle, cf. (25). Enfin, les troisièmes sont dans un autre dialecte encore, et d'une autre époque, à savoir, en bas-navarrais de la seconde moitié du $20^{\mathrm{e}}$ siècle, cf. (26).

(24) J.A. Ubillos (1785, p. 151)

Nori ere barkatzen BAdiozkatzue bekatuak, $t a$ haei

à-qui ere pardonnant $b a$-vous-les-leur(AUX) péchés, et à-eux

barkatuko zaizte, ta nori ere ez BAdiozkatzue barkatzen pardonner-FUT ils-leur-sont et à-qui ere NEG $b a$-AUX pardonnant ta haei ez-zaizte barkatuko.

et à-eux NEG-AUX pardonner-FUT

'A [ceux à] qui vous pardonnerez leurs péchés, ils leur seront pardonnés, et à [ceux

à] qui vous ne les pardonnerez pas, ils ne leur seront pas pardonnés.'

(25) B. Echenique (1857)

a Artakotz, nork ere austen BAdu ${ }^{20}$ manamendu ttipi otarik bat, [...]

pour-cela qui-E ere brisant s'il-AUX commandement petit de-ces un

arras ttikia deitua izain da zeruetako erreinuan;

très petit appelé être-FUT AUX céleste dans-le-royaume

baño nork ere egiten BAdu, eta erakusten BAdu, ura

mais qui-E ere faisant s'il-AUX et montrant s'il-AUX, celui-là

deitua izain da andia zeruetako erreinuan.

appelé être-FUT AUX grand céleste dans-le-royaume

lit. 'Ainsi, celui (qui) s'il viole [=violera] le plus petit de ces commandements sera appelé petit dans le royaume des cieux ; mais celui qui le fera, et

l'enseignera, celui-là sera appelé grand ...'

${ }^{20}$ La version manuscrite de 1855, publiée récemment par Pagola et al. (1995), contient bait-, mais tout le monde s'accorde pour reconnaître que la version imprimée, qui avait été révisée par L.-L. Bonaparte sur place, est plus typique du baztanais d'Elizondo. 
b $[\ldots]$ nork ere iltzen BAdu ${ }^{21}$ geldituko da juiziora obligatua.

(Mt 5,21) qui-E ere tuant s'il-AUX rester-FUT AUX au-jugement soumis lit. 'qui s'il tue, il reste passible du tribunal'

c [...] nor ere aserretzen BAda bere anaiain kontra qui ere se-fâchant s'il-AUX son frère-GEN contre arrozoñik gabe, obligatua izain da juiziora; ... raison sans obligé être-FUT AUX au-jugement lit. 'qui s'il se fâche contre son frère sans raison, il sera passible du tribunal'

d [...] nork ere beatzen BAdu emakume bati ura gutiziatzeko, qui-E ere regardant s'il-AUX femme une-DAT celle-là désirant egiten $\mathrm{du}$ yadanik adulterioa aikin bere biotzean. faisant AUX déjà adultère avec-elle son cœur-LOC lit. 'qui s'il regarde en femme en la désirant, il commet déjà l'adultère dans son cœur.'

(26) D. Soubelet, $(1969, \text { p. 104) })^{22}$

a $\mathrm{Ni}$ niz munduaren argia, nor ere jarraikitzen BAzaut, hura moi je-suis du-monde la-lumière qui ere suivant $b a$-il-me(AUX) lui ez dabila ilhunbetan, bainan ukanen du harek biziko argia. NEG marche dans-la-pénombre mais aura AUX lui de-la-vie la-lumière 'Je suis la lumière du monde: qui me suit ne marche[ra] pas dans les ténèbres, mais aura la lumière de la vie'

b Nork ere ez BAdu onhartzen Jainkoaren erresuma haur ttipi batek qui-E ere NEG ba-AUX recevant de-Dieu le-royaume enfant petit un-E bezala ez da hartan sartuko. comme NEG AUX là entrer-FUT [p. $138=$ Lc $18,17 \&$ Mc 10,15$]$ 'Quiconque n'accueille pas le royaume des cieux comme un petit enfant n'y entrera pas'

Enfin, Lafitte signale explicitement (op. cit., p. 461) que ba- et baitalternent librement dans des phrases concessives que certains appelleraient

${ }^{21}$ Le ms. cité dans la note précédente présente ici une relative semilibre ; il en va de même pour les versets cités en (c) et (d).

${ }^{22}$ Dans un texte du même dialecte mais datant d'un siècle plus tôt, j'ai aussi relevé un emploi de $b a$ - au lieu de bait-, mais maintenant dans une relative (structuralement appositive) introduite par un pronom relatif explicite :

(i) Uroz zerbitzari hura zoina, jiten denean nausia heureux serviteur celui-là qui-SG venant AUX- $n$-LOC le- maître hatzemaiten BAdu hola ari dela

(A. Cazenave, \pm 1850 , Mt 24,46) trouvant ba-AUX ainsi actif qu'il-est

'Heureux le serviteur que son maître, quand il rentre, trouve en train de travailler'

Cet exemple, malheureusement unique à ma connaissance, montre que le schéma logique < conditionnante [ALORS] conditionnée>, que j'ai tenté ici d'appliquer aux PCPC, pourait aussi, au moins dans certains cas (lorsque l'expression nominale est non-référentielle ou non-spécifique), s'appliquer aussi à certaines relatives restrictives. Pour André Rousseau (communication personnelle) l'exemple scolaire bien connu du latin en (b) serait d'ailleurs paraphrasable par : 'Si tu m'as écrit une lettre, elle m'a fait grand plaisir'.

(ii) Quas scripsisti litteras,

que[ACC-FM-PL] tu-écrivis lettre[ACC-FM-PL]

eae mihi joncundissimae fuerunt

elles moi-DAT très-agréables furent

'La lettre que tu as écrite m'a fait grand plaisir.' 
« inconditionnelles » aujourd'hui, comme les suivantes :
(27) Nor bazira / Nor baitzira / qui $b a$-vous-êtes qui bait-vous-êtes 'Qui que vous soyez'
Nor ere baitzira
qui ere bait-vous-êtes

\section{Récapitulation et problèmes en suspens}

6.1. Dans le cas particulier du basque au moins, il semble bien, au vu de la section précédente, que l'on puisse ramener les phrases complexes à corrélatives à une variante morphologique des conditionnelles ${ }^{23}$, dans la mesure où, d'une part, dans les variétés standard de la langue écrite, on a un choix bloqué clair entre deux triplets comprenant un indéfini, un marqueur de subordination, et une conjonction, cf. (28), chaque élément de ces triplets pouvant apparaître comme une variante combinatoire ou distributionnelle de celui de la ligne du dessus ou du dessous, et, d'autre part, dans la mesure où des variétés moins « lissées » de la langue font apparaître certains glissements d'une ligne à l'autre.

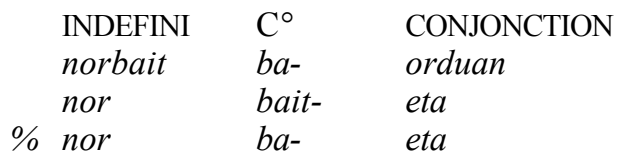

Si cette conclusion est exacte, on peut alors représenter plus précisément les PCPC et les conditionnelles par un même schéma, cf. (29) ${ }^{24}$ :

${ }^{23}$ En tout état de cause, je n'aurai pas seulement pris le contre-pied de la description traditionnelle de Lafitte, loc. cit. dans l'introduction, mais aussi celui de Bittner (2001), qui tente plutôt de ramener les conditionnelles à des structures corrélatives, sur la base du parallélisme de la distribution des préfixes en $j$ - et $t$ - dans des exemples comme les suivants en marathi (empruntés à Andrews (1975)) :

(i) ja mula-ni ja muli-shi dues kela

quel garçon-E quelle fille-GEN haine faisait

tya-ni ti-la mar-li

lui -E elleACC tuer-PASSE

'Quant au garçon et à la fille qu'il haïssait, il l'a tuée.'

(ii) jer to ithe yel, ter miN tya-la mar-in

si lui ici vient alors moi-E lui-ACC tuer-FUT

'S'il vient ici, (alors) je le tuerai.'

${ }^{24} \mathrm{Sur}^{\circ}{ }_{1}$, cf. la remarque concernant le point ' $\mathrm{\square}$ en 2.2 . 


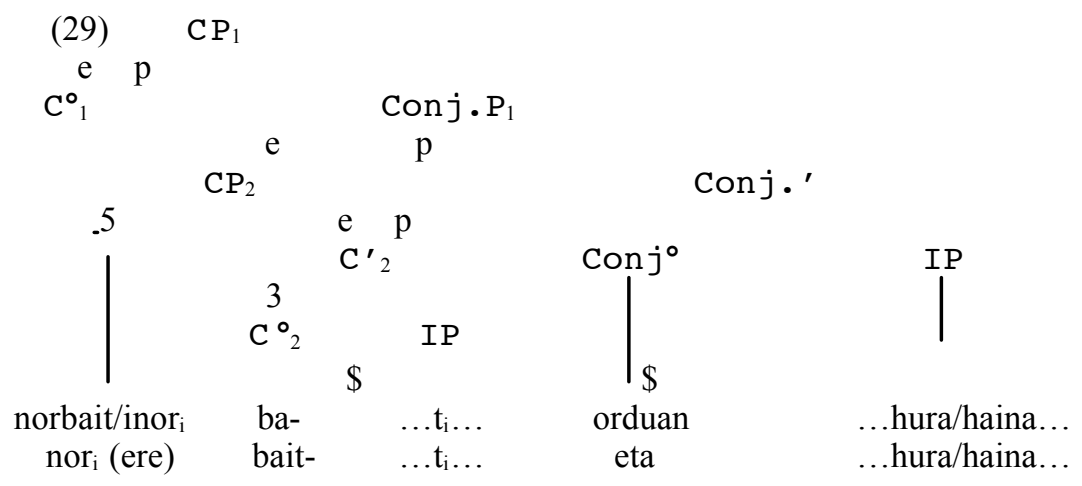

En d'autres termes, tant $b a$ - que bait- occupent la tête $\mathrm{C}^{\circ}$ de la protase, et, par suite, l'indéfini norbait (ou inor) se déplace aussi en syntaxe dite visible, avant s-s / Spell-Out : voir 7.3 pour une réévaluation et l'esquisse d'un développement de cette idée.

6.2. L'analyse présentée ici offre au moins trois avantages. Premièrement, elle est compatible avec la théorie X-barre la plus restrictive qui soit, celle proposée par Kayne (1994), en prenant au sérieux l'idée que la conjonction est en fait la tête fonctionnelle de la phrase complexe : tout recours à l'outil d'adjonction (que Kayne rejette axiomatiquement) est donc inutile. Ensuite, elle permet de se passer en même temps de tout mouvement entre une position interne à l'apodose vers le spécificateur de la conjonction, la protase étant considérée comme étant « générée dans la base » (on pourrait parler de merging dans le jargon du PM) dans cette position, cf. la discussion autour des ex. $(6 a, b)$ : le défaut méthodologique qui est si souvent fait à la théorie de l'antisymétrie de Kayne, à savoir, qu'elle impose non seulement des têtes (fonctionnelles) ad hoc, mais encore des mouvements tout aussi ad hoc, est donc évité. Enfin, cette approche est maximalement compatible avec une démarche compositionnelle concernant l'interface syntaxe-sémantique, dans la mesure où elle permet d'interpréter chaque proposition syntaxique (anglais clauses) comme une proposition logique (angl. proposition), la conjonction se traduisant précisément par le connecteur requis par la quantification, standard ou non, à laquelle l'expression entière est soumise.

A ce sujet, il faut remarquer que la représentation arborescente de (29) est en fait incomplète : il faut également disposer d'une tête quantificationnelle (cf. Beghelli \& Stowell 1995) qui permette à un adverbe comme le 'souvent' de (17b) de prendre l'ensemble de la phrase complexe sous sa portée dès la structure-s (ou Spell-Out) ${ }^{25}$.

${ }^{25}$ Je ne me prononce pas sur ce qui peut se passer en « Forme Logique », à savoir, si cette tête est activée de façon à ce que l'adverbe 'souvent', lorsqu'il se trouve dans l'apodose en s-structure, comme en $(17 \mathrm{a}, \mathrm{c})$ ou $(6 \mathrm{a})$, monte dans son spécificateur pour s'interpréter comme ayant portée sur la structure complexe, ou si une adjonction (mécanisme dont on a vu qu'on pouvait se passer dans la syntaxe dite visible) peut être retenue « après » spell-out (ceci vaut bien sûr également pour la contribution quantificationnelle du pronom labourdin haina, et de l'opérateur générique phoniquement vide que l'on 


\section{Problèmes en suspens}

Pour terminer, je souhaite signaler quelques directions de recherche qui me paraissent pertinentes par rapport aux thèmes abordés dans cet article.

7.1. Tout d'abord, il y a la question de l'étymologie et de la réanalyse morphosyntaxique et sémantique (en synchronie) des morphèmes ou morphes qui entrent en jeu dans les constructions décrites. Considérons plus particulièrement bait- : deux solutions d'égale vraisemblance sont disponibles. Il peut s'agir, bien entendu, du $b a$ - suppositif suivi d'une forme contractée (et bien attestée comme telle) de eta, à savoir, $t a$, ce qui revient à dire qu'il y aurait déjà eu copie de la conjonction introduisant l'apodose sur la tête de la protase.

Mais on peut aussi bien imaginer que le suffixe qui suit $b a$ - serait plutôt le morphème $\boldsymbol{i}$ que l'on trouve par ailleurs dans la seconde série de pronoms indéfinis, celle qui est donnée dans la troisième colonne de (20). En effet, dans les dialectes du Nord, les diphtongues ont tendance à « durcir » les consonnes qui les suivent après une frontière de morphème — par exemple en dévoisant une plosive sonore, comme dans zaiku (ou zauku) 'il nous est', qui s'analyse clairement (et se réalise en guipuzcoan et en basque littéraire unifié) comme zaigu, ou encore en transformant une fricative en affriquée, comme dans zaitzu, zautzu 'il vous est' en face de la forme régulière zaizu. Dans cette perspective, on aurait dans les protases ou bien un indéfini comme nor 'qui', suivi de $b a$-, lui-même suivi de $\boldsymbol{- i}$, ou encore, avec déplacement de ce dernier morphème, la séquence $\boldsymbol{i}+$ nor ... ba.

Quoi qu'il en soit, il est évident qu'une réanalyse revenant à ne plus identifier ces segments comme des morphèmes indépendants a dû se produire très tôt dans l'histoire de la langue, dans la mesure où, comme cela a été rappelé en 2.1, les variétés de basque parlées Outre-Bidassoa ont toujours employé beaucoup plus fréquemment le suffixe complémenteur -en que le préfixe bait- dans dans les protases corrélatives (et dans les relatives formellement appositives), alors que ce suffixe est lui-même totalement opaque ou inanalysable aujourd'hui. Rappelons aussi à cet égard qu'Azkue (loc.cit.) notait que la variante biscayenne très locale baist- de bait- se retrouve tant dans les propositions subordonnées où cet élément fonctionne comme un $\mathrm{C}^{\circ}$ que dans les indéfinis du type norbai(s)t 'quelqu'un'.

7.2. Revenons maintenant sur l'accord indirect que l'on a constaté plus haut entre la conjonction introduisant la proposition principale et l'élément wh- qui spécifie la protase corrélative. On a suggéré plus haut qu'un mécanisme à triple détente était à l'œuvre, l'accord entre la tête conjonctive et son spécificateur conduisant ce dernier, un $\mathrm{CP}$, à recevoir ainsi un trait syntaxique ; dans un second temps, ce trait percolerait vers le $\mathrm{C}^{\circ}$ de la protase ; 
enfin, dans un troisième temps, l'élément $w h$ - hériterait ce trait du $\mathrm{C}^{\circ}$. En fait, on est en droit de se demander s'il n'existerait pas plutôt un mécanisme d'accord entre la conjonction tête et le spécifieur de son spécifieur (l'image miroir du marquage de cas exceptionnel qui permet à une tête d'assigner un cas non pas à son complément structural, mais au spécifieur de ce dernier).

En effet, comme le notait déjà Lafitte (op. cit., p. 105) dans l'exemple repris ci-dessous en (30), il arrive que eta apparaisse entre le mot en wh- et ere (cf. aussi Euskaltzaindia 1999, p. 255, ex. (193)) ${ }^{26}$ :

(30) Non eta ere aurkituko baituzu, han hil zazu où et ere trouver-FUT bait-AUX, là tuer IMPER 'Tuez-le là où vous le trouverez', lit. 'En quelque lieu que vous le trouviez, tuez-le là' (double trad. de Lafitte)

Il est évidemment possible de considérer que le trait syntaxique quel qu'il soit qui est transmis de la tête vers son spécificateur ne puisse se réaliser que dans le spécificateur de ce dernier — où encore comme suffixe sur la protase, comme dans l'ex. suivant, dû à P. Larzabal, un auteur contemporain (cité d'après Euskaltzaindia 1999, p. 255, ex; (192)) :

(31) Lehenago, nork ere pagatzen baitzuen barrika arno gehienik-eta, hura autrefois, qui-E ere payant bait-AUX barrique vin le-plus-eta celui-là zukan pasatzen bozetan.

AUX passant aux-élections

'Jadis, c'était celui qui payait le plus grand nombre de barriques de vin qui gagnait les élections' - lit. 'Qui ere payait le plus de barriques de vin-et, celui-là gagnait'

Cela dit, il faut aussi tenir compte du fait (qui exige une analyse minutieuse que je suis bien incapable de faire pour le moment) que eta n'est pas simplement déplacé, mais peut être copié ou dupliqué, lorsque les phrases complexes à corrélatives sont des comparatives de degré. En voici quelques exemples guipuzcoans, empruntés à un recueil récent de proverbes (Zavala 1985, vol. 2) ${ }^{27}$.

${ }^{26}$ A vrai dire, il semble que ce tour soit surtout fréquent lorsque la protase s'interprète comme une circonstancielle de temps, comme en (i) :

(i) Noiz eta ere ikusi bainuen [...], tiro batez hil nuen. (Lafitte 1962, p. 459)

quand et ere vu bait-AUX coup de feu d'un tué AUX

Quand je le vis..., je le tuai d'un coup de fusil.'

Inutile de le dire, les rapports syntaxiques (qui semblent évidents) et sémantiques entre les PCPC et les phrases complexes à circonstancielle spécifique disloquée à gauche (comme c'est le cas ici) demandent aussi à être approfondis.

${ }^{27}$ On notera au passage, trois fois sur quatre, la présence de la conjonction $\operatorname{ordu}(a) n$ 'alors' qui caractérise « normalement » les apodoses conditionnées. Il significatif qu'inversement, dans certaines langues, le «même » morphème puisse être employé soit pour coordonner deux éléments (en ce qui nous concerne, deux propositions [syntaxiques]), soit pour associer une protase conditionnante et une apodose conditionnée : c'est par exemple le cas du swahili, 
(32) a Zenbat eta geiago izan, gero ta geiago nai combien et plus avoir après et plus vouloir 'Plus on a (et) plus on veut'

b Zenbat eta geiago, orduan ta naiago combien et plus alors et plus-volontiers (id.)

(33) Zenbat eta obia, ordun ta okerr[ag]o combien et meilleur, alors et pire

'Meilleur c'est, pire c'est' (cf. plus ça change, plus c'est la même chose')

(34) Zenbat eta zarr[ag]o, ordun ta berro plus et plus-vieux alors eta plus-nécessiteux [berro = bearrago] 'Plus on vieillit, plus on est dans le besoin'

Evidemment, l'interprétation minimaliste actuelle, qui revient à postuler que tout déplacement est une copie suivie d'un effacement, peut être invoquée, mais le blocage même de l'effacement de eta dans l'apodose reste problématique. $^{28}$

7.3. Revenons enfin aux conditionnelles. Le fait que, dans ces phrases complexes, le mot baldin puisse se trouver en tête de la subordonnée conditionnante, et être en quelque sorte repris par $b a-$, cf. (35b), indique peut-être que plusieurs têtes fonctionnelles sont en jeu, ce que semble corroborer l'existence du morphème complexe balinba-, cf. (35c), ce mot s'analysant alors comme la forme contractée résultant de l'incorporation de $b a$-à baldin, opération qui aurait à son tour pour conséquence la montée de l'indéfini :

(35) a Norbait / Inor etortzen ba.da quelqu'un/anybody venant si AUX 'Si quelqu'un / qui que ce soit vient'

b Baldin norbait / inor etortzen ba.da (id.)

c Norbait / Inor etortzen balinba.da (id.)

Par parité de raisonnement, on serait alors en droit de réinterpréter le ere des protases corrélatives (cf. la note 16) comme étant en fait le correspondant corrélatif du baldin conditionnant, ere forçant la montée de l'élément wh-dans son spécificateur, et baldin, par contre, le bloquant. Mais cette remarque demande, bien entendu, un travail technique qui reste à faire.

En tout cas, un dernier agument en faveur de la thèse centrale de cet article, à savoir, que les PCPC sont des variantes de conditionnelles, est encore fourni par les très nombreux allomorphes et variantes dialectales de baldin, qui incorporent toutes d'une manière ou d'une autre la conjonction eta : baldin-eta, baldinetari, baldinetaria, baldinetarik, baldinetariak, baldinetarian... (cf. Michelena et al. 1989, pp. 788-791), phénomène qui pourrait s'expliquer par les mécanismes d'échange de traits discutés en 7.2.

mais c'est aussi le cas de l'ancien français, cf. Rebuschi (sous p.).

${ }^{28}$ De même que son placement à droite de gero ou orduan, quelle que soit la machinerie syntaxique adoptée. 


\section{Bibliographie}

\section{Sources basques}

ASTARLOA, Pedro J. (1816-18) : Urteco Domeca gustijetaraco verbaldi icasibidecuac [...]. [Cité in Arejita (1978).]

CAZENAVE [Casenave], $( \pm 1860)$ : Jesu Khristoin Ebanjelio Seidia San Mathiuïn aäbera. Ms., publié in R. M. Pagola et al. (eds.), BOE, Ekialdeko Behe-nafarrera, I, I (Bilbao, Deustuko Unibertsitatea, 1999), 17-76.

CHOURIO, M. (1720) : Jesu-Christoren Imitacionea [...] Escararat itçulia. Rééd., Bayonne, Trebos, 1788; facsim.: Hordago-Lur, Saint-Sébastien, 1979.

DUVOISIN, Jean (1859-65) : Bible edo Testament Zahar eta Berria [...]. Londres. Facsim., Bilbao, Gran Enciclopedia Vasca, 1972.

ECHENIQUE, B. (1855) : Ed. du ms. d'Echenique (1857) in R. M. Pagola et al. (eds.), BOE, Iparraldeko Goi-Goinafarrera, I (Bilbao, Deustuko Unibertsitatea, 1995), 97-176.

ECHENIQUE, B. (1857) : Evangelio según San Mateo. Londres. Rééd. en facsim. in L.L. Bonaparte, Opera Omnia Vasconice, II (Bilbao, Euskaltzaindia), 91-150.

ETCHePARE, B. (1545), Lingvee Vasconvm Primitice. Ed. par P. Altuna, Bilbao, Mensajero, 1980.

HARANEDER, Joannes. (1740) : Jesu Christoren Evangelio Saindua. Ms., éd. par P. Altuna, Bilbao, Euskaltzaindia, 1990.

HARRIET, Maurice (1855) : Iesu-Christo gure Iaunaren Testament Berria [...]. Bayonne: E. Lasserre.

ISASTI $( \pm 1625)$ : Proverbios en Basquence por abecedario. Ms, éd. in L. Michelena, Textos arcaicos vascos (Madrid, Minotauro, 1964), 3.3.3.

Itun Berria (1983) : Bilbao, Bibli Elkarte Batuak / PAX Argitaletxea.

LEON, L. (1929) : Jesu-Kristoren Imitazionea. Turnhout, Brepols.

LEON, L. (1947) : Jesu-Kristo gure Jaunaren Ebanjelio Saindua. Ustaritz, chez l'auteur.

LARZABAL, Piarres. \pm 1980 . Orrega. [Cité in Euskaltzaindia (1999).]

LIZARRAGA, Joaquín. $( \pm 1800)$ : Urteko igande guzietarako prediku laburrak.Ms., éd. par F. Ondarra, Bilbao, Euskaltzaindia (1990).

LIZARRAGA, Joaquín. (1868) : Jesucristoren Evangelio Saindua, Juanec dacarran guisara. Londres. Rééd. fac-sim., OOV III, 13-102, \& éd. du ms. dans BOE, Hegoaldeko Goi-nafarrera IV (1996), 1235-1275.

POUVREAU, S. 1669. Iesusen Imitacionea. Paris. Rééd.: Hordago-Lur, SaintSébastien, 1979.

Refranes y sentencias. 1596. Ms., anon., éd. par J. A. Lakarra, Bilbao, Euskaltzaindia, 1996.

SOUBELET, Domingo (1969) : Jesus. Imprimé à Ligugé (Vienne), Presses d'Aubin.

UBILlOS, Juan Antonio (1785) : Christau doctriñ berri-ecarlea [...]. Tolosa. Rééd. facsim. avec Intro. Par P. Altuna, Bilbao, Euskaltzaindia (1989).

ZAVALA, Antonio. 1985. Esaera Zaararen bilduma berria I, II. Tolosa: Auspoa, \#184 \& \#185.

\section{Etudes grammaticales et linguistiques}

ALTUBE, Seber. 1929. Erderismos.Euskera 10, 1-4. Rééd. facsim., Bilbao : Indauchu, 1975.

ANDREWS, Avery. 1975. Studies in the Syntax of Relative and Comparative 
Constructions. Thèse de doctorat : MIT.

AREJITA, Adolfo. 1978. Euskal Joskera. Durango : Zugaza.

AZKUE, Resurrección Ma . 1923-25. Morfología Vasca. Gramática básica dialectal del Euskera. Rééd. facsim., Bilbao : Editorial La Gran Enciclopedia Vasca,1969.

BEGHELLI, Filippo, \& STOWELL, Tim. 1997. 'Distributivity and Negation'. In A.. Szabolcsi (éd.), Ways of Scope Taking (Dordrecht : Kluwer), 71-107.

BITTNER, Maria. 2000. 'Nominal and Modal Referents in Correlatives'. Communication à la Table ronde sur la syntaxe et la sémantique des constructions relatives, Tel Aviv, juin.

CHENG, Lisa L.-S. 1995. 'On Dou-Quantification'. Journal of East Asian Linguistics 4.3, 197-234.

CHOMSKY, Noam. 1977. 'On Wh-Movement'. In P. Culicover, T. Wasow \& A. Akmajian (eds.),Formal Syntax (New York: Academic Press), 71-132.

EUSKALTZAINDIA. 1999. Euskal gramatika; lehen urratsak, 5: Mendeko perpausak, 1. Bilbao : Euskaltzaindia.

GROSU, Alexander. 2000. 'Type-Resolution in relative constructions: featural marking and dependency encoding'. In A. Alexiadou et al. (eds.), The Syntax of Relative Clauses (Amsterdam: Benjamins), 83-119.

GROSU, Alexander, \& LANDMAN, Fred. 1998. 'Strange Relatives of the Third Kind'. Natural Language Semantics 6.2, 125-170.

LAFITTE, Pierre. 1962. Grammaire basque (navarro-labourdin littéraire). Bayonne: Amis du Musée Basque \& Ikas.

LIPTAK, Anikó. 2000. 'Multiple Relatives as Relatives of Questions'. In G. Alberti \& I. Kenesei (eds.), Approaches to Hungarian, VII : Papers from the Pécs Conference (Szeged : JATE Press), 151-177.

MiCHELENA, Luis, et al. 1989. Diccionario General Vasco / Orotariko Euskal Hiztegia, vol. III (Ase-Bapuru). Bilbao : Euskaltzaindia, Desclée de Brouwer \& Mensajero.

Partee, B. 1975. 'Montague Grammar and Transformational Grammar', Linguistic Inquiry 6.2, 203-300.

REBUSCHI, Georges. 1997. 'Remarques sur le pronom haina'. Lapurdum 2, 63-81.

REBUSCHI, Georges. 1998. 'Nouvelles remarques sur haina'. Lapurdum 3, 5375.

REBUSCHI, Georges. 1999. 'Types de langues de types de constructions : le cas des corrélatives'. In A. Söres \& C. Marchello-Nizia (eds.), Typologie des langues, universaux linguistiques [Univ. Paris $\mathrm{X}:$ LINX, numéro spécial], 55-72.

REBUSCHI, Georges. [sous presse]. 'Towards a Theoretical Treatment of LeftDislocated "Subordinate" Clauses'. In: Proceedings of the Bayonne Colloquium in Pierre Lafitte's memory.

RIZZI, Luigi. 1997. 'The Fine Structure of the Left Periphery'. In L. Haegeman (éd.), Elements of Grammar; Handbook of Generative Syntax (Dordrecht : Kluwer), 281-337.

SRIVASTAV, Veneeta. 1991. 'The Syntax and Semantics of Correlatives'. Natural Language and LinguisticTheory 9.4, 637-686.

$$
* * * * *
$$

<rebuschi@idf.ext.jussieu.fr> 